\title{
BIÓPSIA CEREBRAL ESTEREOTÁXICA EM PACIENTES COM AIDS COM SINTOMAS NEUROLÓGICOS
}

\author{
JOSÉ AUGUSTO NASSER*, CARLOS IVAM CONFORT**, ANDREI FERRAZ**, \\ JOSÉ CARLOS ESPERANÇA***, FRANCISCO DUARTE****
}

\begin{abstract}
RESUMO - Estudo prospectivo mostrando a importância da biópsia cerebral estereotáxica no manuseio do paciente com AIDS e que apresenta sintomatologia neurológica confirmada através de imagens. Todos estes pacientes obedecem a escalonamento terapêutico e, depois de terem sido dados os passos prévios, a biópsia passa a ser indicada visando ao diagnóstico etiológico adequado. O protocolo foi iniciado em agosto-1995 e concluído em dezembro-1996. Vinte pacientes foram biopsiados. Nosso protocolo é semelhante ao descrito por Levy e col. (Chicago IL, USA). Linfoma primário foi o diagnóstico predominante, seguido de leucoencefalopatia multifocal progressiva, toxoplasmose e leucoencefalopatia pelo HIV. Um caso de linfoma de células gigantes diplóico foi incluído. Nossa morbi/mortalidade foi nula. Por esta amostragem é possível concluir que a biópsia cerebral estereotáxica é um procedimento seguro e eficaz em pacientes com AIDS.
\end{abstract}

PALAVRAS-CHAVE: biópsia cerebral estereotáxica, síndrome da imunodeficiência adquirida, linfoma, toxoplasmose, leucoencefalopatia multifocal progressiva, encefalopatia pelo HIV.

\section{Image guided stereotactic brain biopsy in AIDS patients with neurological manifestations}

ABSTRACT - Prospective series showing the importance of computerized stereotactic brain biopsy in the management of AIDS patients neurologically symptomatic and confirmed by images. Patients undergone an algorithm step by step done by their own doctors and referred to us for stereotactic biopsy. Our protocol was opened in August 1995 and closed in December 1996. Twenty patients were biopsied. This protocol is similar to the Levy's one (Chicago IL, USA). We have got diagnosis in all cases. Lymphoma was predominant and followed by toxoplasmosis, progressive multifocal leukoencephalopathy and HIV encephalopathy. We included one patient with diploic giant cells lymphoma. Our mortality and morbidity was zero. By these results we conclude that stereotactic biopsy in AIDS patients is safe and effective.

KEY WORDS: stereotactic brain biopsy, acquired immunodeficiency syndrome, lymphoma, toxoplasmosis, progressive multifocal leukoencephalopathy, HIV encephalopathy.

Este estudo é o resultado de toda uma avaliação de pacientes com AIDS que, em seu curso de doença, apresentaram sintomas neurológicos. A partir deste instante, inicia-se a investigação através da aplicação de um algoritmo, visando identificar e tratar corretamente a lesão que está levando a este problema. Para isto, no diagnóstico por imagens a tomografia computadorizada do crânio (TC) e a ressonância magnética (RM) são imperativas, dando início ao protocolo de investigação. Este nosso protocolo é semelhante ao descrito por Levy e col. da Northwestern University (Chicago IL, USA $)^{1,2}$. Desta forma foi possível conduzir o estudo de maneira coerente e sistematizada, proporcionando a perfeita análise e comparação de várias séries.

* Mestrando em Neurocirurgia (UNIFESP - Escola Paulista de Medicina, São Paulo - SP), Neurolife Laboratórios, Estereolife Neurocirurgia Funcional e Estereotáxica (NLL/ELNFE); ** NLL/ELNFE, Hospital Universitário Clementino Fraga Filho da Faculdade de Medicina da Universidade Federal do Rio de Janeiro (UFRJ); ***Professor Adjunto da Anatomia Patológica da FM/UFRJ; ****Professor Titular da Anatomia Patológica da FM/UFRJ e Pesquisador do CNPq. Aceite: 12-janeiro-1998.

Dr. José Augusto Nasser - Rua do Russel 450/802 - 22210-010 Rio de Janeiro RJ - Brasil. 
O nosso foco dentro deste protocolo é a importância da biópsia cerebral estereotáxica computadorizada (BE) para o estabelecimento de etiologia de lesão que já tenha sido amplamente investigada e está frente ao inconclusivo. Dessa maneira é possível estabelecer com eficiência e segurança para o paciente, por vezes muito debilitado, o diagnóstico preciso da doença ${ }^{3-7}$. Para que este procedimento tenha o sucesso almejado, o trabalho em equipe multidisciplinar é imperativo, contando com: neurorradiologista, neurocirurgião estereotáxico e neuropatologista. A presença destes profissionais em sala, discutindo caso a caso, resulta na eficácia do diagnóstico.

\section{METODOLOGIA}

\section{Material clínico}

São analisados 20 pacientes com AIDS e com sintomas neurológicos, submetidos a todos os passos do escalonamento terapêutico (Quadro 1), no período de agosto-1995 a dezembro-1996.

\section{Algoritmo}

Observando o Quadro 1, estes pacientes neurologicamente sintomáticos foram submetidos a estudos de imagem (TC com duplo contraste e RM com contraste). Se os estudos fossem normais ou mostrassem apenas atrofia, o exame de líquor era realizado; caso este fosse normal, o paciente era orientado a frequentar o serviço mensalmente. Quando se observassem lesões múltiplas do sistema nervoso central (SNC), um teste terapêutico para toxoplasmose era iniciado e monitorado por duas semanas com imagem; se houvesse regressão das lesões o tratamento seria intensificado, caso contrário a BE estaria indicada. Para as lesões solitárias e para pacientes instáveis neurologicamente, a BE era indicada prontamente.

\section{Procedimento cirúrgico}

Em todos os casos nós usamos o Sistema Esterotáxico ETM-03B (Micromar, Diadema SP) e o programa msa.out para o cálculo das coordenadas (Armando Alaminos Bouza). O halo é fixado sob anestesia local (lidocaína a $2 \%$ ). Imediatamente após, o paciente é levado para o Centro de Imagens onde realizamos a estereotomografia do crânio com contraste venoso. Todas as coordenadas são enviadas a central de trabalho (Epson- 4SLC) que,

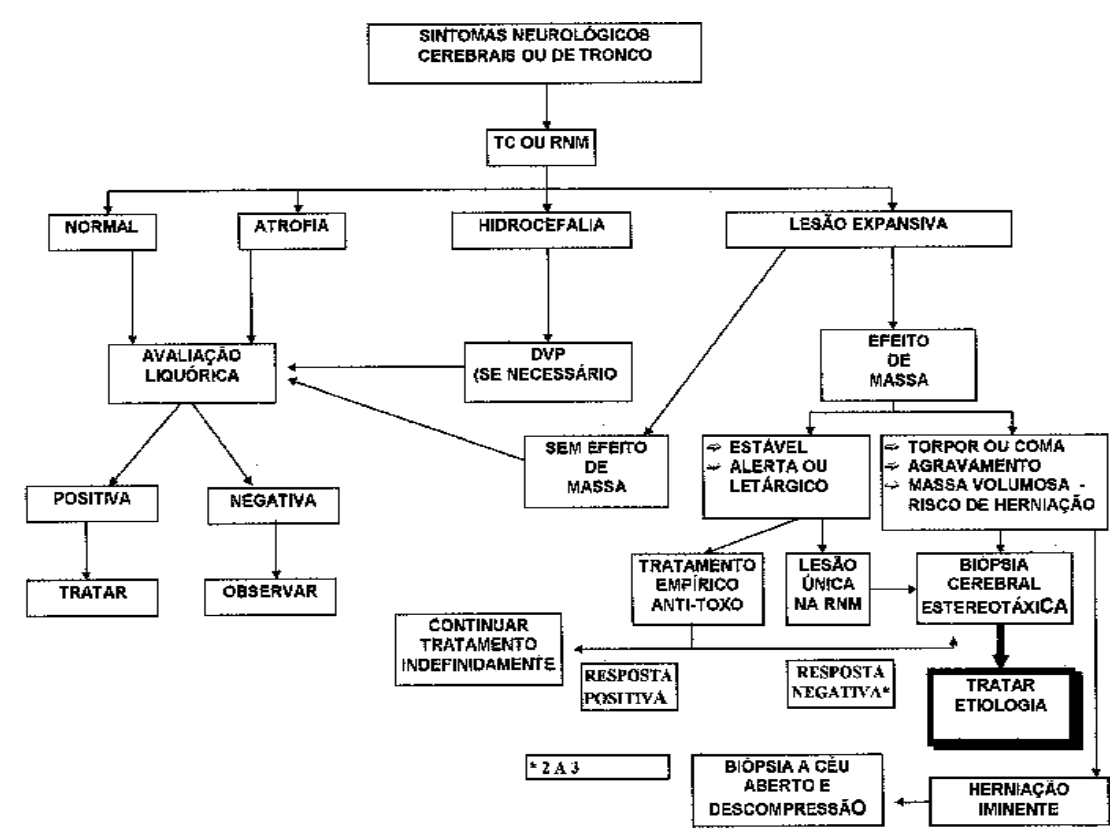

Quadro 1. Escalonamento terapêutico de pacientes com AIDS com sintomas neurológicos. 
através do programa msa.out, permite obter todas as coordenadas dos alvos eloquentes, identificados pelos exames de imagem. O paciente é então levado ao Centro Cirúrgico. Sob sedação (Propofol) e anestesia local, usando novamente lidocaína a $2 \%$, é feita a incisão com os dados fornecidos pelo programa e aplicados ao sistema estereotáxico. Uma trepanação no ângulo adequado é realizada,seguida da abertura dural. Podemos fazer então hemostasia sob magnificação, evitando complicações. O passo seguinte, com todo o sistema revisto, é a introdução da agulha tipo janela lateral Sedan ${ }^{6}$ ou Gildemberg ${ }^{7}$. O material coletado é examinado pelo neuropatologista simultaneamente. Todos os alvos eloquentes são biopsiados na maioria das vezes. Ocasionalmente o diagnóstico já pode ser dado em sala, outras vezes apenas a positividade do material é evidenciada e o diagnóstico estabelecido posteriormente em preparados na parafina. De qualquer forma, todas as amostras são exaustivamente analisadas e submetidas, se necessário, a bateria de técnicas seletivas e imuno-histoquímicas. Os pacientes habitualmente permanecem no hospital por 24 horas.

\section{Histopatologia}

O material, recebido a fresco durante o ato cirúrgico é colocado sobre superfície de vidro, filiforme e medindo cerca de $1 \mathrm{~cm}$ é estirado, retirando-se mínimos fragmentos das duas pontas, das quais são confeccionados esfregaços com auxílio de lâmina de bisturi. Estes são imersos imediatamente em etanol absoluto por um minuto. A parte restante do material retirado é colocada em solução fixadora de formol a $10 \%$. Então, as lâminas já fixadas, são hidratadas em etanol em concentrações decrescentes (a 90\%; a 80\%; a 70\%; água); coradas em hematoxilina de Harris por um minuto; desidratadas em alcoois crescentes (70\%; 80\% e absoluto-duas vezes); clarificadas em xilol (duas vezes); montadas com resina acrílica e lamínula. O material fixado em formol a $10 \%$ (mínimo de 5 horas) é incluído em parafina; os blocos são cortados e corados em hematoxilina/eosina e eventualmente submetidos as técnicas seletivas, de acordo com as necessidades de cada caso ${ }^{8}$.

\section{RESULTADOS}

Vinte pacientes preencheram os critérios de indicação e foram biopsiados no período mencionado (Tabela 1). A média de idade foi 36 anos. Os locais variaram, sendo: 18 supratentoriais, 1 infratentorial e 1 calota craniana. Todos os pacientes, neste período, obtiveram o diagnóstico definitivo de suas lesões. Houve predomínio do linfoma primário (35\%) (Fig 1), seguido de toxoplasmose (20\%), leucoencefalopatia pelo HIV (20\%), leucoencefalopatia multifocal progressiva (20\%) (Fig 3) e, um caso de linfoma de células gigantes (5\%).

Cumpre ressaltar a inclusão, nesta série, desse paciente, com múltiplas lesões diplóicas que infiltravam a dura subjacente. Foi feita marcação estereotáxica e ressecção guiada da lesão. O diagnóstico definitivo foi linfoma de células gigantes. Seu estudo de imagem mimificava mieloma múltiplo.

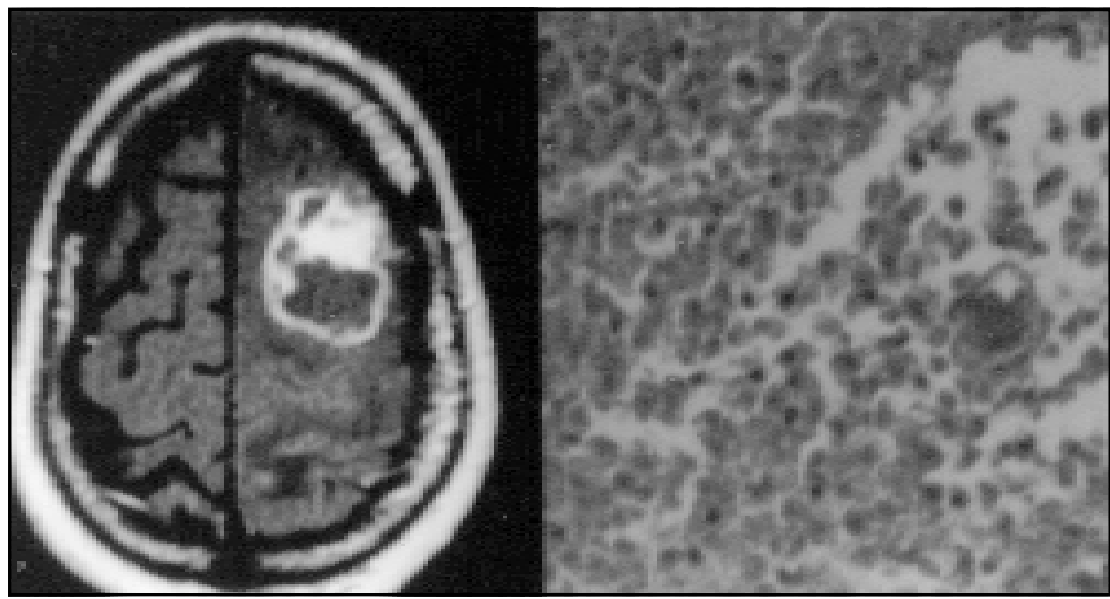

Fig 1. À esquerda, ressonância magnética do cérebro mostrando lesão anelar com captação periférica e heterogênea do contraste paramagnético, necrose central, situada no córtex frontal esquerdo. À direita, a histologia da biópsia estereotáxica confirmando o linfoma primário do SNC. 
Tabela 1. Pacientes submetidos à biópsia estereotáxica no período de agosto-1995 a dezembro-1996.

\begin{tabular}{|c|c|c|c|c|}
\hline Identificação & Idade & LCR prévio & $\mathrm{TC} / \mathrm{RM}$ & Histologia \\
\hline W.P. & 46 & $\begin{array}{c}\text { HIV + } \\
\text { Toxoplasmose }\end{array}$ & $\begin{array}{l}\text { Lesão talâmica esq com efeito de massa } \\
\text { captante de contraste periférico } \\
\text { Sequela de toxoplasmose }\end{array}$ & Linfoma primário do $\mathrm{SNC}$ \\
\hline J.A.C. & 42 & $\mathrm{HIV}+$ & $\begin{array}{l}\text { Lesão hiperintensa em T2 em corona } \\
\text { radiada bilateral }\end{array}$ & Leucoencefalopatia pelo HIV \\
\hline R.S.C. & 28 & $\mathrm{HIV}+$ & $\begin{array}{l}\text { Lesão talâmica esq com captação anelar } \\
\text { de contraste e efeito de massa }\end{array}$ & $\begin{array}{l}\text { Processo inflamatório crônico } \\
\text { necrosante }\end{array}$ \\
\hline A.L.S. & 47 & $\mathrm{HIV}+$ & $\begin{array}{l}\text { Lesão frontal de substância branca (SB) } \\
\text { bilateral acometendo o corpo caloso } \\
\text { Sequela de toxoplasmose }\end{array}$ & Linfoma primário do SNC \\
\hline J.C.S. & 36 & $\mathrm{HIV}+$ & $\begin{array}{l}\text { Três lesões de SB } \\
\text { (corona radiada bilateral) }\end{array}$ & Leucoencefalopatia pelo HIV \\
\hline M.V.D. & 32 & $\begin{array}{l}\mathrm{HIV}+ \\
\text { toxoplasmose }\end{array}$ & $\begin{array}{l}\text { Lesões difusas em núcleos basais } \\
\text { bilaterais, com quebra de barreira, edema } \\
\text { e intensa captação de contraste }\end{array}$ & Toxoplasmose \\
\hline J.A.M.S. & 31 & $\mathrm{HIV}+$ & $\begin{array}{l}\text { Lesão captante de contraste difusamente } \\
\text { no núcleo lentiforme esq }\end{array}$ & Toxoplasmose \\
\hline R.R.R. & 33 & $\mathrm{HIV}+$ & $\begin{array}{l}\text { Lesão extensa acometendo todo o lobo } \\
\text { fronto-parietal esq com captação } \\
\text { periférica de contraste }\end{array}$ & Linfoma primário do SNC \\
\hline O.C.N. & 42 & $\mathrm{HIV}+$ & $\begin{array}{l}\text { Múltiplas lesões diplóicas líticas difusa, } \\
\text { algumas com extensão para a dura máter, } \\
\text { captante de contraste }\end{array}$ & Linfoma de células gigantes \\
\hline N.M.A. & 48 & HIV+ & $\begin{array}{l}\text { Lesões difusas na corona radiada e SB } \\
\text { periventricular hipointensas em } \mathrm{T} 2 \text { e em } \\
\text { densidade protônica }\end{array}$ & $\begin{array}{l}\text { Leucoencefalopatia } \\
\text { multifocal progressiva }\end{array}$ \\
\hline A.F.C. & 42 & $\mathrm{HIV}+$ & $\begin{array}{l}\text { Lesões expansivas frontobasais dir e } \\
\text { putaminal dir }\end{array}$ & Linfoma primário do SNC \\
\hline M.D.Q. & 33 & HIV+ & $\begin{array}{l}\text { Lesão hipointensas em T1 e hiperintensas } \\
\text { em T2 bifrontal (SB) e parieto-occipital } \\
\text { dir }\end{array}$ & $\begin{array}{l}\text { Leucoencefalopatia } \\
\text { multifocal progressiva }\end{array}$ \\
\hline J.G.P.S. & 35 & HIV+ & $\begin{array}{l}\text { Lesão expansiva talâmica dir com } \\
\text { captação anelar de contraste }\end{array}$ & Linfoma primário do $\mathrm{SNC}$ \\
\hline W.S. & 32 & HIV+ & $\begin{array}{l}\text { Lesão expansiva hipodensa com pouca } \\
\text { captação de contraste talâmica dir }\end{array}$ & Toxoplasmose \\
\hline T.C.L.S. & 33 & HIV+ & $\begin{array}{l}\text { Lesão de SB bifrontal e corona radiada } \\
\text { bilateral hiperintensas em } \mathrm{T} 2 \text { e DP }\end{array}$ & Leucoencefalopatia pelo HIV \\
\hline R.C.M. & 35 & $\mathrm{HIV}+$ & $\begin{array}{l}\text { Lesão de SB em corona radiada bilateral } \\
\text { e periventricular }\end{array}$ & Leucoencefalopatia pelo HIV \\
\hline A.C.C. & 28 & $\begin{array}{l}\text { HIV+ } \\
\text { toxoplasmose }\end{array}$ & $\begin{array}{l}\text { Lesão talâmica esq com halo periférico } \\
\text { captante de contraste na TC }\end{array}$ & Linfoma primário do $\mathrm{SNC}$ \\
\hline A.D.F. & 23 & $\begin{array}{l}\text { aumento de } \\
\text { proteína }\end{array}$ & $\begin{array}{l}\text { Lesão cerebelar direita hiperintensa em } \\
\text { T2 e DP }\end{array}$ & Leucoencefalopatia pelo HIV \\
\hline M.V.D.C. & 32 & HIV+ & Lesão no cúneos a esq & Linfoma primário do $\mathrm{SNC}$ \\
\hline S.C.M. & 37 & HIV+ & $\begin{array}{l}\text { Lesões em núcleos basais bilaterais } \\
\text { captantes de contraste }\end{array}$ & $\begin{array}{l}\text { Leucoencefalopatia } \\
\text { multifocal progressiva }\end{array}$ \\
\hline
\end{tabular}

Idade em anos; LCR, líquor; esq, esquerda; dir, direita; SB, susbtância branca. 


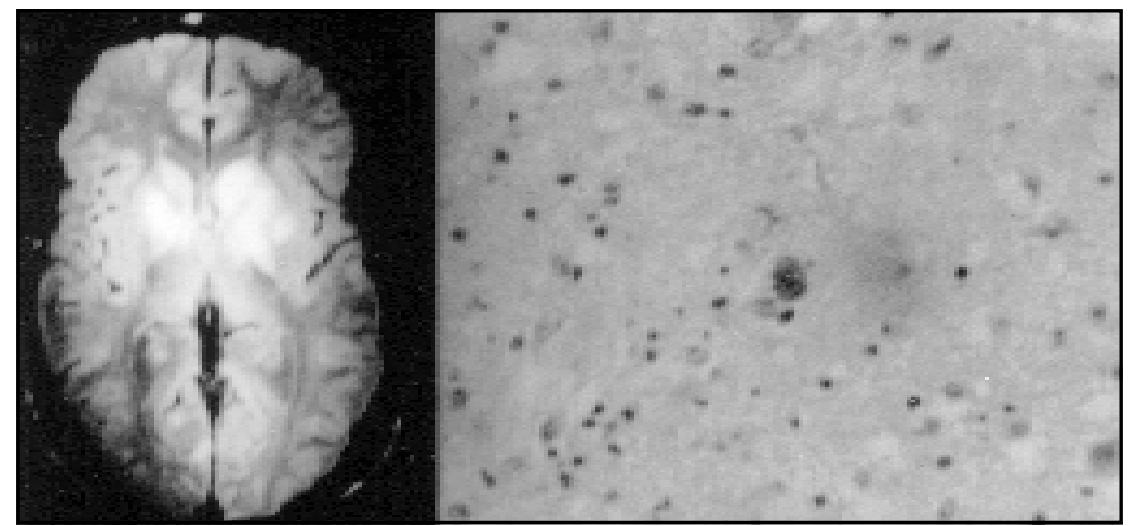

Fig 2. À esquerda, ressonância magnética do cérebro em densidade protônica, mostrando lesão difusa bilateral acometendo os núcleos: caudado, globo pálido e putamen bilateralmente e braço posterior da cápsula interna bilateralmente. À direita, histologia da biópsia estereotáxica identificando o parasita no centro e confirmando neurotoxoplasmose.

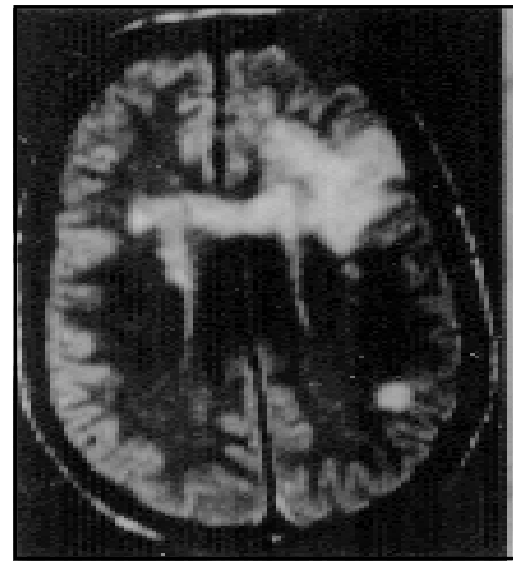

Fig 3. À esquerda, ressonância magnética do cérebro em T2, mostrando lesão infiltrativa de substância branca no lobo frontal esquerdo, invadindo o lobo frontal direito através do joelho do corpo caloso. À direita, histologia da biópsia estereotáxica mostrando rarefação axonal e a inclusão característica da leucoencefalopatia multifocal progressiva.

A morbidade e a mortalidade foram nulas.

Três casos mostraram, no estudo liquórico, positividade para toxoplasmose. Desta forma foram tratados por 3 semanas e reavaliados em RM. Como não obtiveram melhora foram submetidos a BE. Dois apresentavam linfoma primário e o terceiro, toxoplasmose refratária ao tratamento convencional (Fig 2).

\section{DISCUSSÃO}

A BE, nos pacientes com AIDS e com sintomas neurológicos, é procedimento seguro e desde os primeiros estudos de Levy já se demonstrava a importância no diagnóstico e na orientação da terapêutica apropriada ${ }^{1}$. A equipe multidisplinar é imperativa para a positividade das amostras ${ }^{8}$. $\mathrm{O}$ uso adequado do equipamento de estereotaxia e sistema computadorizado acoplado, com programas especiais de análise de imagens e mapeamento das lesões, possibilita o acesso seguro a lesões situadas 
em qualquer parte do cérebro ou tronco cerebral ${ }^{7,9-16}$. A obtenção de biópsias seriadas nos dá uma idéia global da lesão, pois, nas lesões expansivas é frequente a necrose central ${ }^{2,7,17}$. No caso das lesões tumorais, é importante obter fragmentos que estejam nos limites entre o tecido normal e o tumoral. Outro fator determinante, e que se torna imperativo neste projeto, é a presença em sala de neuropatologista experiente no assunto. A discussão amostra por amostra, entre neurocirurgião e neuropatologista em sala, foi responsável pela positividade em todos estes casos.

Na maioria das séries, existe certo equilíbrio entre linfoma, toxoplasmose e leucoencefalopatia multifocal progressiva. Isto difere da nossa amostragem até o momento. Com número maior de pacientes, será possível verificar se isto se mantém. Quanto à morbidade e mortalidade, nossa casuística está de acordo com a literatura. É sabido da possibilidade de sangramento em linfoma, pela fragilidade e abundância vascular; certamente pode ter ocorrido em nossos casos, porém em nenhum deles foi evidenciada sintomatologia ${ }^{1,2,5}$.

A utilização do escalonamento diagnóstico e terapêutico, descrito por Levy e col. ${ }^{1,2}$, veio auxiliar e sistematizar de forma importante os pacientes com AIDS, pois $20 \%$ dos pacientes, ainda sem diagnóstico, apresentam sinais e sintomas de acometimento do SNC. Cerca de $40 \%$ dos pacientes com AIDS apresentam sinais e sintomas de doença do SNC, quando feito o diagnóstico; 70 a 90\% dos pacientes com AIDS apresentam doença do SNC durante o curso da doença; finalmente, 80 a $100 \%$ apresentam alterações anátomo-patológicas do SNC à necrópsia $(50 \%$ com mais de uma doença e 5 a $10 \%$ com processos expansivos, com predomínio de neurotoxoplasmose) $)^{3,4,10,17,18}$.

Nós concluimos que a biópsia cerebral estereotáxica em pacientes com AIDS com sintomas neurológicos é procedimento seguro e importante que, muitas vezes, vem auxiliar o médico solicitante no diagnóstico etiológico e no tratamento específico destas patologias. É importante salientar o progresso do tratamento moderno desta doença, que vem aumentando consideravelmente a sobrevida dos pacientes.

\section{REFERÊNCIAS}

1. Levy RM, Pons VG, Rosemblum ML. Central Nervous mass lesions in the AIDS. J Neurosurg 1984;61:9-16.

2. Levy RM, Russel E. Yungbluth, Hidvegi DF, Brody BA, Canto MC. The efficacy of image guided stereotactic brain biopsy in neurologically symptomatic AIDS patients. Neurosurgery 1992;30:186-190.

3. Cescato VAS. Biópsia estereotáxica de lesões encefálicas inflamatórias In Machado LR, Nóbrega JPS, Livramento JP, Spina-França A. Neuroinfecção 94. São Paulo. Clínica Neurológica HC/FMUSP, 1994:249-252.

4. Chappel ET, Guthrie BL, Orestein J. The role of stereotactic biopsy in the management of HIV-related focal brain lesions. Neurosurgery 1992;30:825-829.

5. Prayson RA, Estes ML. Stereotactic brain biopsy for diagnosis of PML. South Mel J 1993;69:1381-1394.

6. Sedan R, Peragut JC, Vallicioni P. Presentation d'un appareillage original pour biopsie cerebrale et tumorale en conditions stereotaxiques. Comunication a la Societe de Neurochirurgie de Langue Francaise, Dec 1975.

7. Kelly PJ. Tumor stereotaxis. Philadelphia: WB Sauders, 1991.

8. Berkeley BB, Adams JH, Doyle D, Graham DI, Harper CG. The smear technique in the diagnosis of neurosurgical biopsies. N Z Med J 1978;87:12-15.

9. Apuzzo MLJ, Sabshin JK. Computed tomographic guidance stereotaxis in management of intracranial mass lesions. Neurosurgery 1983;12:277-285.

10. Cescato VAS, Teixeira MJ. Biópsia estereotáxica. In Nitrini R, Machado LR. Condutas em neurologia 1993. São Paulo. Clínica Neurológica HC/FMUSP, 1993:179-184.

11. Clarke RH. Investigations of the central nervous system: methods and instruments. Jonhs Hopkins Hospital Report, 1920; Special Volume: 1-162.

12. Daumas-Duport C, Monsaingeon V, Szenthez, Seiklag. Serial stereoactic biopsies a double histological code of gliomas according to malignancy and 3-D configuration as an aid to therapeutic decision and assesment of results. Appl Neurophysiol 1982;45:431-437.

13. Kondziolka D, Dempsey PK, Lunsford LD, Kestle JRW, Dolan EJ, Kanal. A comparison between MRI and CT for stereotactic coordinate determination. Neurosurgery 1992;30:402-407.

14. Lobato RD, Rivas JJ, Cabello A. Stereotactic biopsy of brain lesions visualized with CT. Appl Neurophysiol 1982;45:426-430.

15. Ostertag CB, Mennel HD, Kiessling M. Stereotactic biopsy of brain tumors. Surg Neurol 1980;14:275-283.

16. Thomas DGT, Kitchen ND. Stereotactic techiniques for brain biopsies. Arch Dis Child 1993;69:621-624.

17. Schlitt MJ, Morawetz, Bonnin JM, Zeiger HE, Whitlety RJ. Brain biopsy for encephalitis. Clin Neurosurg 1986;33:591-602.

18. Friedman WA, Sceats DJ Jr, Nestok BR. The incidence of unexpected pathological findings in an image-guided biopsy series: a review of 100 consecutive cases. Neurosurgery 1989;25:180-184. 